\title{
Difficulties in Translating Conjunctions in Arabic and English Languages: Study Based on Translation Students of South Eastern University of Sri Lanka
}

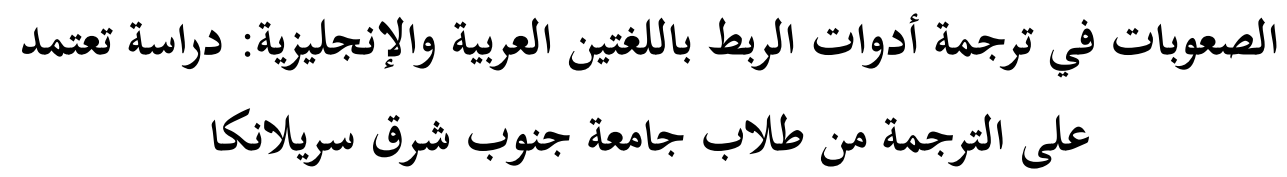

N. M. Mohammed Rizvi

rizvihaleemi@gmail.com

Department of Arabic Language

South Eastern University, Sri Lanka

Munas M.H.A

munasmha@gmail.com

Department of Arabic Language

South Eastern University, Sri Lanka

Iqbal Saujan

Department of Islamic Studies

South Eastern University, Sri Lanka

- Received: 29.08.2021 • Accepted: 18.10.2021 • Published online: 26.11.2021

Abstract: Each language has its own set of rules. Based on this, the rule of conjunctions in the Arabic language differs from the English language, because they come from two separate language families. Thus, the learners of Arabic and English languages as a foreign language face difficulty and commit mistakes in identifying meanings of conjunctions. Therefore, this Study focuses on identifying difficulties when translating. This study employed a descriptive and contrastive methodology. The data were gathered from interviews and questionnaire that were distributed to all specialization Students in linguistic and translation from South Eastern University of Sri Lanka. MS Excel Software was used for the data analysis. The findings show, the students face difficulties in determining appropriate conjunctions and understanding their meaning, using suitable words. Based on the researcher's findings, continuous training in translation with caring of grammatical errors is recommended. This research will help foreign language learners, translators and students.

Keywords: conjunctions, difficulties, translation students, Arabic, English 
N. M. Mohammed Rizvi, Munas M.H.A, Iqbal Saujan

الملخص: كل لغة لها مجموعة القواعد الخاصة بها. وبناءً على ذلك ، فإن قاعدة الاقتران في اللغة العربية تختلف عن اللغة الإنجليزية ، لأنها تأتي من عائلتين لغويتين منفصلتين. وبالتالي ، يواجه متعلمي اللغتين العربية والإنجليزية كلغة أجنبية صعوبة ويرتكبون أخطاء في تحديد معاني الاقتران. لذلك ، تركز هذه الدراسة على تحديد الصعوبات عند الترجمة.

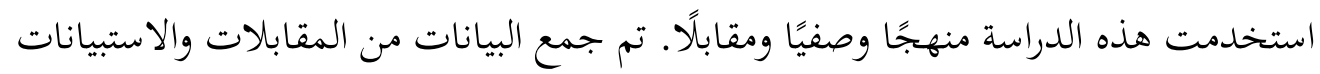
التي تم توزيعها على جميع طلاب التخصص في اللغويات والترجمة من جامعة جنوب شرق

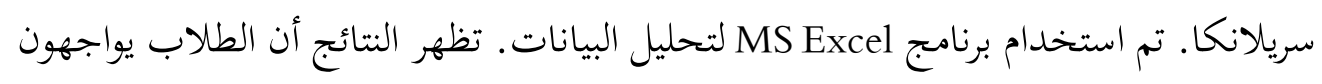

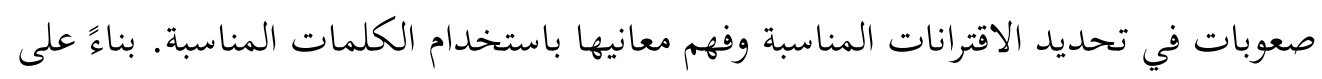
نتائج الباحث ، يوصى بالتدريب المستمر في الترجمة مع مراعاة الأخطاء النحوية. سيساعد

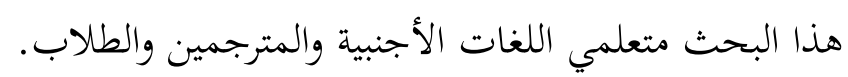
كلمات دلالية: اقتران ، صعوبات ، ترجمة طلابية ، عربي ، إنجليزي

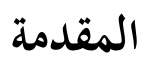

إن اللغة العربية هى لغة القرآن الكريم ولها فضل واسع وشرف مستمر من بداية

نزول القرآن المجيد على خاتم الأنبياء محمد صلى الله عليه وسلم. وهى عنوان

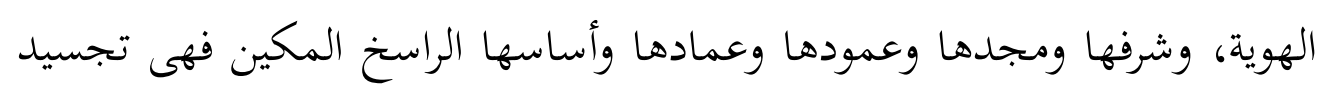
للهوية الإسلامية التي شرفها الله وأنزل بها قرآنه الكريم ووسيلة حفظه وانتشاره بين الناس فهي اللغة الخالدة إلى أن يرث الله الأرض ومن عليها. تعتبر اللغة العربية من أمهات اللغات المنتشرة حول العالم، وهى اللغة التي يتحدث بها أكثر من ملياري إنسان على سطح الأرض، كما أن اللغة العربية هي لغة الصيغ والإعراب والتصرف الته كما تختص بغنية أصواتها واشتقاق كلماتها ومتزاحمة فصاحتها متصفة بالمرونة 
N. M. Mohammed Rizvi, Munas M.H.A, Iqbal Saujan

والتنوع في بناء جملها، مع أن كل حرف في اللغة العربية له قيمة وكل حركة لها هدف خاص.

وأما اللغة الإنجليزية فهي أيضا من أمهات اللغات المهمة الرسمية حول العالم. وهي تستخدم بمعظم سكان العالم من مغاربه ومشارقه. ولها صيغ خاصة في بنائها وأساليب منظمة باستخدام صحيح. وهتان اللغتان الرئيستان تتضمنان عدة صيغ في بناء الجمل الصحيحة ومنها أدوات العطف. هذه الأدوات تهتم في نظم العبارات والفقرات بلا خطء من ناحية بنية النحو. تسمى أدوات العطف "Conjunctions" في اللغة الإنجليزية. واستخدمت هذه الأدوات لأداء دور معين في تركيب الجمل ولذا يقال لها بالأدوات لأنها تربط الجمل والكلمات معا لمساعدتها على التناغم والترابط وإيصال المعنى المراد، بحيث تحتاج الجمل في اللغة العربية عند تكوينها والوصل بينها إلى روابط حتى يأتي التعبر عن الجملة صحيحا، فلهذا تستخدم أدوات العطف لتوازن بين الجمل أو الفقرات، أو عند الدخول إلى فكرة أو موضوع جديد، أو لتعليل وجهة نظر ما وتوضيحها، أو عقد مقارنة بين شيئين والإختيار بينهما، وغيرهما من المهام الأخرى التي تستوجب الاستعانة بأدوات العطف. إن الطلبة المتخصصين في مجال اللسانيات والترجمة يتوجهون عوائق ومصاعب عند ترجمة أدوات العطف من العربية إلى الإنجليزية أو من الإنجليزية إلى العربية بسبب أن هذه أدوات العطف تختلف استخداماتها بين هتين اللغتين العربية والإنجليزية باختلاف الأسر اللغوية التي تتضمن هتين اللغتين. ولذا هذا البحث يسعى على معرفة ماهية الصعوبات عند ترجمة أدوات العطف بين اللغتين العربية والإنجليزية تحت عنوان " صعوبات ترجمة أدوات العطف في اللغتين العربية والإنجليزية ". 
N. M. Mohammed Rizvi, Munas M.H.A, Iqbal Saujan

إن أكثر الطلبة المتخصصين في مجال اللسانيات والترجمة يواجهون الصعوبات في ترجمة أدوات العطف في الجمل والعبارات وفي تحديد معانيها المناسبة فلذلك تتغير المعاني والأفكار الأصلية في لغة الهدف. وهكذا أنهم لا يستخدمون أدوات العطف استخداما صحيحا في الجمل والعبارات عند عملية الترجمة. لقد اعتنى الباحثون في دراساتهم بكثير من الموضوعات المتعلقة بأدوات العطف؛ لمساعدتها على التناغم والترابط وإيصال المعنى المراد. ولكن هذا البحث يتميز من ناحية ترجمة أدوات العطف بين اللغتين العربية والإنجليزية وهكذا يساعد دارسي مجال اللسانيات والترجمة لإتقان فن الترجمة بين اللغتين العربية والإنجليزية كما يدل على استخدام أدوات العطف عند عملية الترجمة استخداما صحيحا. منهجية البحث

تستخدم هذه الدراسة المنهج الوصفي والتقابلي لتحليل المعلومات الأولية والثانوية. أما المعلومات فقد أخذها الباحث من هذه الطرق الآتية: الإستبانة - يسعى الباحث على اطلاع صعوبات ترجمة أدوات العطف وفي اختيارها من الطلبة المتخصصين في مجال اللسانيات والترجمة من خلال الإستبانة. المقابلة - تكمل هذه الأداة مع الطلاب المتخصصين في مجال اللسانيات والترجمة بأسلوب حواري. طريقة تحليل المعلومات من حيث المنهج التقابلي بمساعدة استخدام البرنامج SPSS software نتائج الدراسة وتحليلها 
N. M. Mohammed Rizvi, Munas M.H.A, Iqbal Saujan

يشتمل ذلك على ثلاث خطوات لبناء الإستبانة المتمثلة في قياس الصعوبات التي يواجهها دارسوا اللغة العربية لغة أجنبية في تحديد معاني أدوات العطف وترجمتها بين اللغتين العربية والإنجليزية. تكونت الإستبانة من اثنا عشر سؤالا، ويدور منها ثمانية أسئلة عن المعلومات الشخصية للمشارك والمفاهيم العامة عن أدوات العطف بين اللغتين العربية والإنجليزية، والسؤال التاسع يحتوي على خمس ولى جمل من اللغة العربية وهكذا السؤال العاشر يتضمن خمسة أسئلة لتحديد الصعوبات عند ترجمة الجمل العربية إلى اللغة الإنجليزية، أما السؤال الحادي عشر فهو يتكون من خمس جمل من اللغة الإنجليزية وهكذا السؤال الثاني عشر يتضمن خمسة أسئلة لتحديد الصعوبات عند ترجمة الجمل الإنجليزية إلى اللغة العربية. يتناول هذه الدراسة تحليل نتائج الدراسة الميدانية من خلال إجابات أفراد مجتمع الدراسة للإستبانة التي تتكون من اثنا عشر سؤالا تحت المحاور الثلاث : محور تحديد مدى المفاهيم العامية المتعلقة بأدوات العطف وترجمتها بين اللغتين العربية والإنجليزية، محور ترجمة الجمل العربية إلى اللغة الإنجليزية مع الإشارة إلى لى الصعوبات عند ترجمتها، محور ترجمة الجمل الإنجليزية إلى اللغة العربية مع الإشارة إلى الصعوبات عند ترجمتها، وتعالج باستخدام مفاهيم الإحصاء الوصفي وأساليبه الإحصائية وصولا إلى النتائج وتفسيرها كما يلي: السؤال الأول : جنس أفراد مجتمع العينة

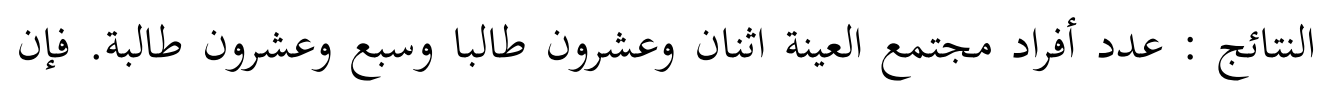
من الأفراد 45 \% ذكورا و 55 \% إناثا، ويبينها الجدول رقم (2.1 )، والشكل (2.1). الجدول 2.1 بيان الجنس 
N. M. Mohammed Rizvi, Munas M.H.A, Iqbal Saujan

\begin{tabular}{|c|c|c|c|}
\hline النسبة المثوية & العلدد & الجنس & ر. \\
\hline$\% 45$ & 22 & الذكر & 1 \\
\hline$\% 55$ & 27 & الأنثى & 2 \\
\hline$\% 100$ & 49 & المجموع & \\
\hline
\end{tabular}

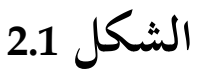

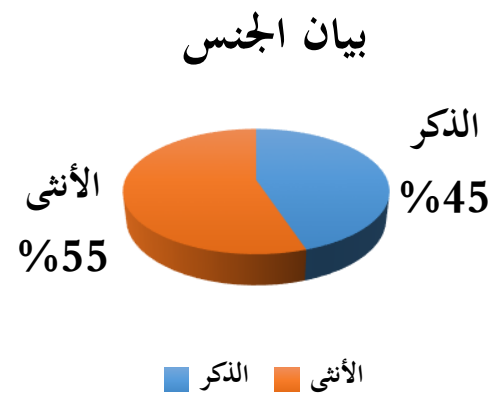

السؤال الثاني : السنة الدراسية

النتائج : عدد أفراد مجتمع العينة تسعة وأربعون طالبا. أن الأغلبية منهم من السنة الرابعة، فأما الأقلية منهم من السنة الثانية، ويبين ذلك الجدول رقم (2.2) ، والشكل

$$
\text { الجدول } 2.2 \text { العينة الدراسية }
$$

\begin{tabular}{|c|c|c|c|}
\hline النسبة المئوية & العدد & السنة الدراسية & م.ر \\
\hline$\% 33$ & 16 & السنة الثانية & 1 \\
\hline$\% 22$ & 11 & السنة الثالثة & 2 \\
\hline
\end{tabular}


N. M. Mohammed Rizvi, Munas M.H.A, Iqbal Saujan

\begin{tabular}{llll}
\hline \% 45 & 22 & 3 \\
\hline$\% 100$ & 49 & المجنة الربعوع & \\
\hline
\end{tabular}

\section{2}

\section{السنة الدراسية}

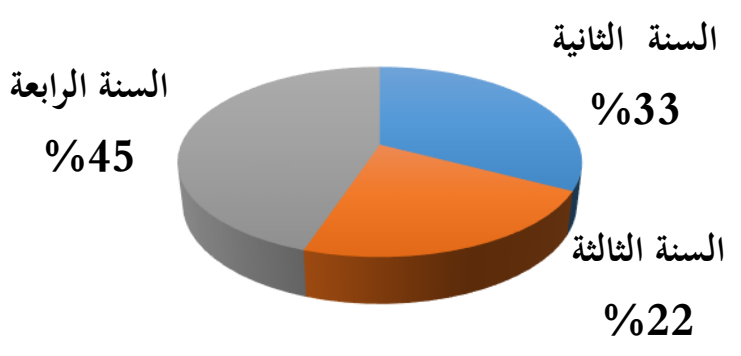

السؤال الثالث: أريد أن أدرس اللغة العربية

النتائج : وبالنظر إلى درجة إرادة تعلم اللغة العربية أن الأغلبية وافقوا تعليم اللغة العربية تماما وفي حين وافق عشرون. ولكن أي منهم لم يحايدوا ولم يرفضوا تماما أيضا. وتؤيد هذه النتائج أن الطلبة يريدون تعلم اللغة العربية. ويوضحها الجدول رقم(2.3)،

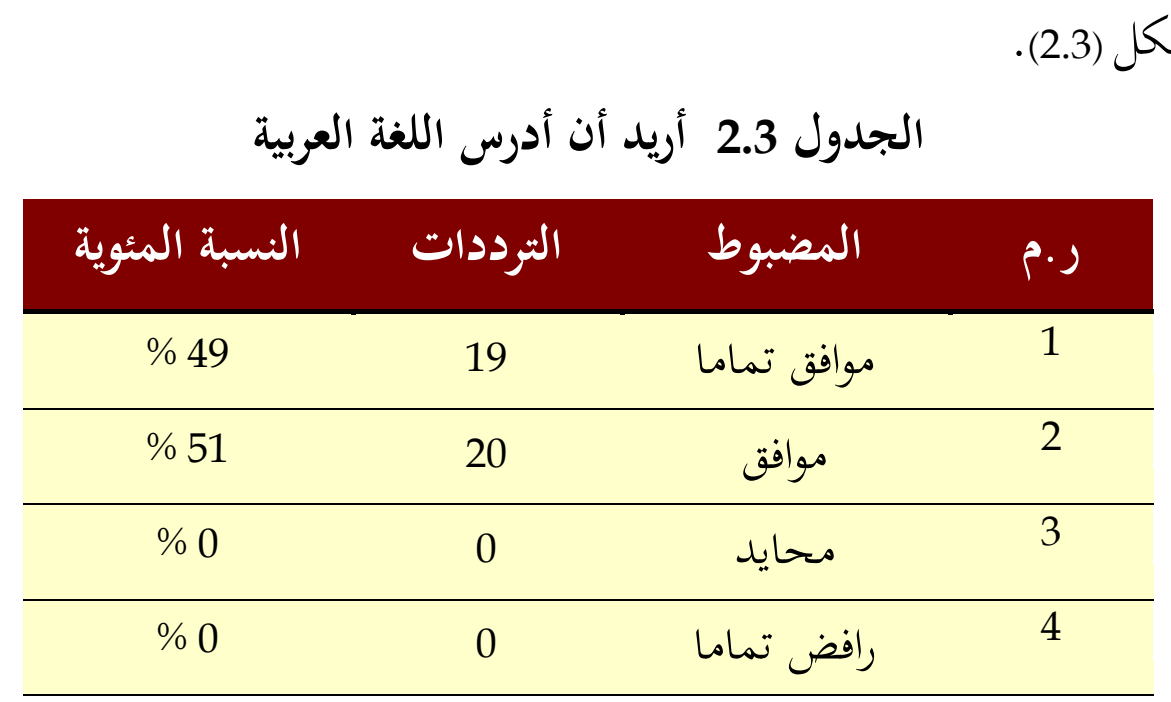


N. M. Mohammed Rizvi, Munas M.H.A, Iqbal Saujan

\begin{tabular}{lll}
\hline المجموع 100 & 49 \\
\hline
\end{tabular}

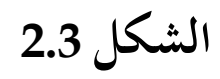

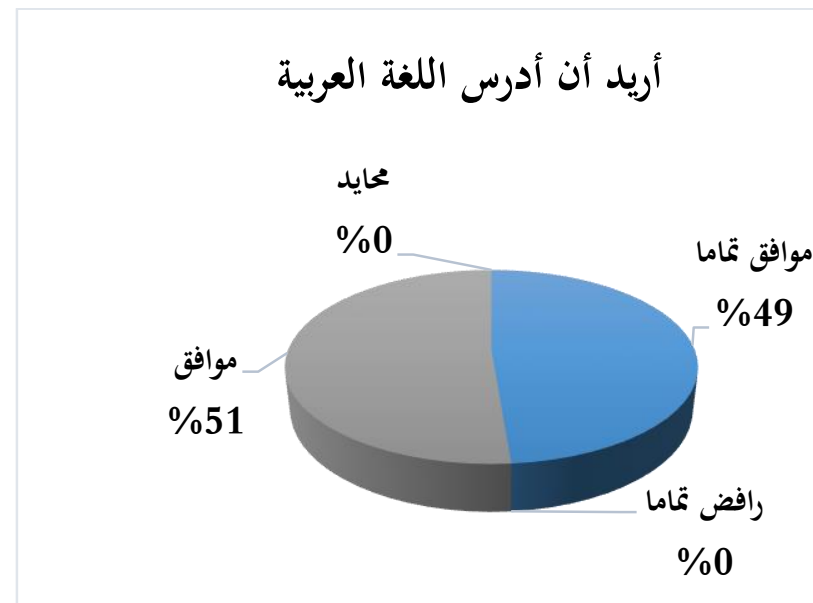

السؤال الرابع: أنا متعلق بعملية الترجمة

النتائج : وبالنظر إلى درجة علاقة الأفراد بعملية الترجمة توجد الأغلبية وافقوا فيه

وفي حين، 39 \% وافقوا تماما وأما الأقلية منهم فتوسطوا فيه. ولكن أي منهم لم يرفضوا تماما. وتؤيد هذه النتائج أن الطلبة يتعلقون بعملية الترجمة. ويوضحها الجدول رقم(2.4)، والشكل (2.4).

الجدول 2.4 أنا متعلق بعملية الترجمة

\begin{tabular}{|c|c|c|c|}
\hline النسبة المئوية & الترددات & المضبوط & م.J \\
\hline$\% 35$ & 17 & موافق تماما & 1 \\
\hline$\% 39$ & 19 & موافق & 2 \\
\hline$\% 26$ & 13 & محايد & 3 \\
\hline$\% 0$ & 0 & رافض تماما & 4 \\
\hline
\end{tabular}


N. M. Mohammed Rizvi, Munas M.H.A, Iqbal Saujan

\begin{tabular}{lll}
\hline \% 100 & 49 & \\
& & المجموع \\
\hline
\end{tabular}

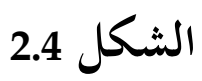

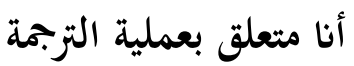

رافض تماما

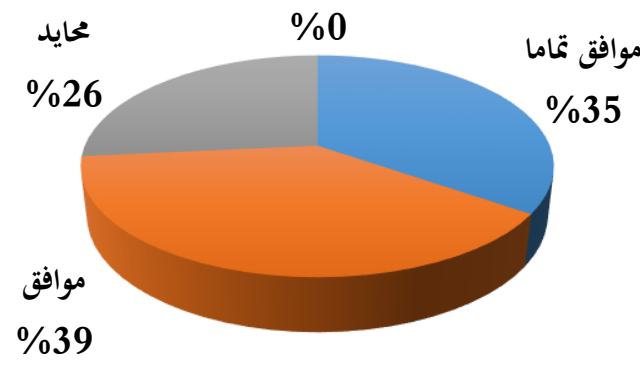

السؤال الخامس : أواجه الصعوبات عند الترجمة من اللغة الإنجليزية إلى اللغة العربية النتائج : وبالنظر إلى الصعوبات عند الترجمة من اللغة الإنجليزية إلى اللغة العربية نجد أن الأغلبية؛ اثنان وعشرون مشاركا وافقوا وجود الصعوبات فيها وفي حين، أربعة عشر مشاركا وافقوا تماما فيها، وأما الأقلية منهم ثلاثة عشر مشاركا رفضوا تماما. ولكن أي منهم لم يحايدوا وجود الصعوبات فيها. وتؤيد هذه النتائج أن الطلبة يواجهون الصعوبات عند ترجمة اللغة الإنجليزية إلى اللغة العربية بدرجة متوسطة. ويوضحها الجدول رقم(2.5)، والشكل (2.5). الجدول 2.5 الصعوبات عند الترجمة من اللغة الإنجليزية إلى اللغة العربية

\begin{tabular}{|c|c|c|}
\hline النسبة المئوبية & الترددات & المضبوط \\
\hline$\% 29$ & 14 & موافق تماما \\
\hline
\end{tabular}


N. M. Mohammed Rizvi, Munas M.H.A, Iqbal Saujan

\begin{tabular}{|c|c|c|c|}
\hline$\% 45$ & 22 & موافق & 2 \\
\hline$\% 0$ & 0 & محايد & 3 \\
\hline$\% 26$ & 13 & رافض تماما & 4 \\
\hline$\% 100$ & 49 & المجموع & \\
\hline
\end{tabular}

الصعوبات عند الترجمة من اللغة الإنجليزية إلى اللغة العربية

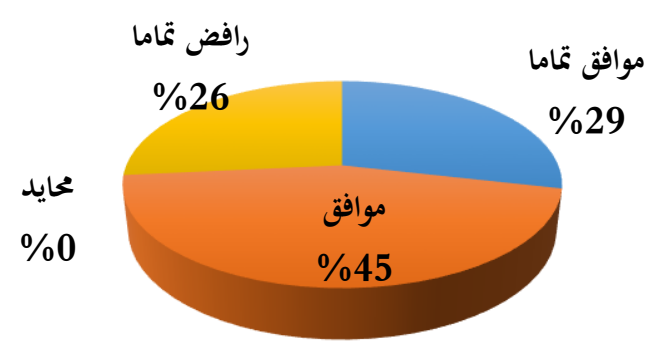

السؤال السادس: أواجه الصعوبات عند الترجمة من اللغة العربية إلى اللغة الإنجليزية النتائج: وبالنظر إلى الصعوبات عند الترجمة من اللغة العربية إلى اللغة الإنجليزية

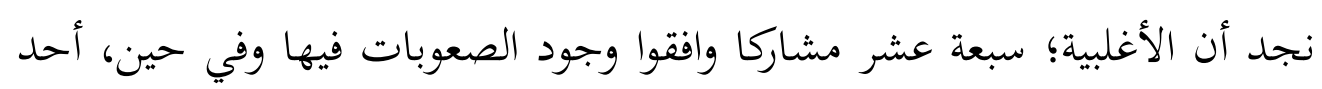
عشر مشاركا وافقوا فيها تماما وعشرة توسطوا فيها. ولكن عشرة منهم رفضوا تماما وجود الصعوبات فيها. وتؤيد هذه النتائج أن الطلبة يواجهون الصعوبات عند الترجمة من اللغة العربية إلى اللغة الإنجليزية بدرجة متوسطة. ويوضحها الجدول رقم(2.6)؛ والشكل (2.6). الجدول 2.6 الصعوبات عند الترجمة من اللغة العربية إلى اللغة الإنجليزية 
N. M. Mohammed Rizvi, Munas M.H.A, Iqbal Saujan

\begin{tabular}{|c|c|c|c|}
\hline النسبة المئوية & الترددات & المضبوط & ر. \\
\hline$\% 23$ & 11 & موافق تماما & 1 \\
\hline$\% 35$ & 17 & موافق & 2 \\
\hline$\% 22$ & 11 & محايد & 3 \\
\hline$\% 20$ & 10 & رافض تماما & 4 \\
\hline$\% 100$ & 49 & المجمو ع & \\
\hline
\end{tabular}

الصعوبات عند الترجمة من اللغة العربية إلى اللغة الإنجليزية

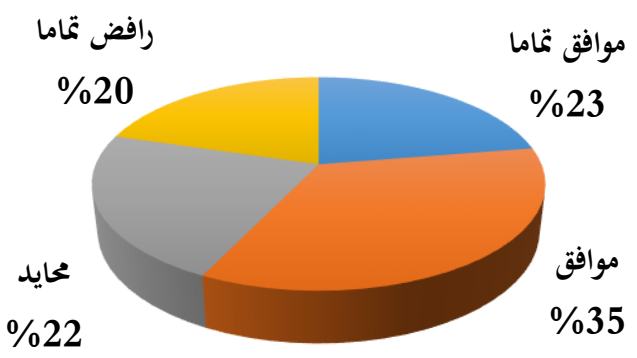

السؤال السابع : أعرف عن قواعد استخدام أدوات العطف في اللغة العربية النتائج: وبالنظر إلى درجة وجود المعرفة عن قواعد استخدام أدوات العطف في اللغة العربية نجد أن 43 \% مشاركا وافقوا وجود المعرفة فيه وفي حين، خمسة عشر مشاركا وافقوا فيها تماما وأما الأقلية منهم ثلاثة عشر مشاركا توسطوا فيها. ولكن أي منهم لم يرفضوا وجود المعرفة فيه. وتؤيد هذه النتائج أن الطلبة يعرفون عن قواعد استخدام 
N. M. Mohammed Rizvi, Munas M.H.A, Iqbal Saujan

أدوات العطف في اللغة العربية بدرجة متوسطة. ويوضحها الجدول رقم(2.7)،

والشكل (2.7).

الجدول 2.7 معرفة عن قواعد استخدام أدوات العطف في اللغة العربية

\begin{tabular}{|c|c|c|c|}
\hline النسبة المئوية & الترددات & المضبوط & ر. \\
\hline$\% 31$ & 15 & موافق تماما & 1 \\
\hline$\% 43$ & 21 & موافق & 2 \\
\hline$\% 26$ & 13 & محايد & 3 \\
\hline$\% 0$ & 0 & رافض تماما & 4 \\
\hline$\% 100$ & 49 & المجموع & \\
\hline
\end{tabular}

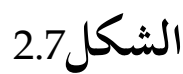

معرفة عن قواعد إستخدام أدوات العطف في اللغة العربية

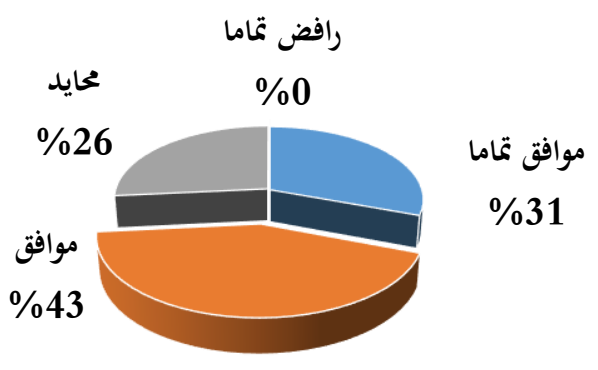

السؤال الثامن: أعرف عن قواعد استخدام أدوات العطف في اللغة الإنجليزية النتائج : وبالنظر إلى درجة وجود المعرفة عن قواعد استخدام أدوات العطف في اللغة الإنجليزية نجد أن الأغلبية من المشاركين وافقوا وجود المعرفة فيه وفي حين، 
N. M. Mohammed Rizvi, Munas M.H.A, Iqbal Saujan

ستة عشر مشاركا وافقوا فيها تماما، وخمسة عشر مشاركا توسطوا فيها. ولكن أي منهم لم يرفضوا وجود المعرفة فيه. وتؤيد هذه النتائج أن الطلبة يعرفون عن قاعدة أدوات العطف في اللغة الإنجليزية بدرجة متوسطة. ويوضحها الجدول رقم(2.8)،

والشكل (2.8) (2.8) (2) (2)

الجدول 2.8 معرفة عن قواعد استخدام أدوات العطف في اللغة الإنجليزية

\begin{tabular}{|c|c|c|c|}
\hline النسبة المئوية & الترددات & المضبوط & ر. \\
\hline$\% 33$ & 16 & موافق تماما & 1 \\
\hline$\% 37$ & 18 & موافق & 2 \\
\hline$\% 30$ & 15 & محايد & 3 \\
\hline$\% 0$ & 0 & رافض تماما & 4 \\
\hline$\% 100$ & 49 & المجموع & \\
\hline
\end{tabular}

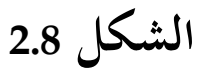

معرفة عن قواعد إستخدام أدوات العطف في اللغة الإنجليزية رافض تماما

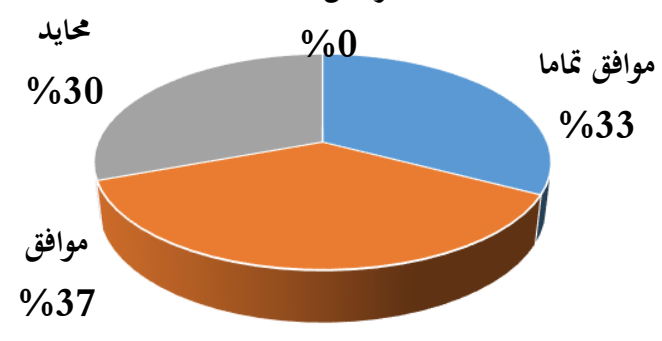


N. M. Mohammed Rizvi, Munas M.H.A, Iqbal Saujan

السؤال التاسع : هو يتضمن خمس جمل عربية للترجمة إلى اللغة الإنجليزية. ومن هذه الجمل، تحدد أخطاء الطلبة. وهي: أنا لا أحب القهوة ولكن أنا مولع بالشاي. اندم فيعفو لك. الله يبدأ الخلق ثم يعيده. هل هذه الهدية لي أم لأحدٍ آخر؟ يُه أكلت برتقالاً لا تفاحة.

ترجم الأغلبية من الطلبة الجملة الأولى صحيحا في درجة أعلى، وأما الأقلية منهم فأخطأوا في قواعد النحوية وثمانية في عناية نظام الجملة الإنجليزية، وأربعة عشر في تحديد أدوات العطف المناسبة، وستة في استخدام الكلمات الإنجليزية المناسبة للكلمات العربية. وترجم الأغلبية من الطلبة الجملة الثانية صحيحا في درجة

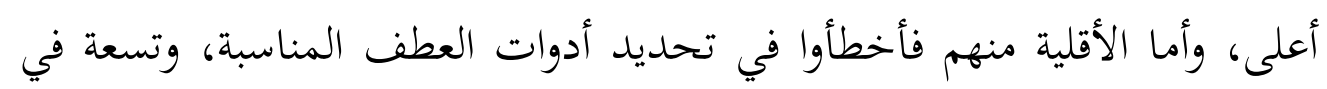
عناية نظام الجملة الإنجليزية، وأحد عشر في قواعد النحوية، وثمانية في استخدام الكلمات الإنجليزية المناسبة للكلمات العربية.

وترجم الأغلبية من الطلبة الجملة الثالثة صحيحا في درجة أعلى، وأما الأقلية منهم فأخطأوا في قواعد النحوية، واثنا عشر في عناية نظام الجملة الإنجليزية، وثمانية في تحديد أدوات العطف المناسبة، وستة في استخدام الكلمات الإنجليزية المناسبة للكلمات العربية. وترجم الأغلبية من الطلبة الجملة الرابعة صحيحا في درجة أعلى، وأما الأقلية منهم فأخطأوا في استخدام الكلمات الإنجليزية المناسبة للكلمات العربية، وخمسة في قواعد النحوية، وتسعة في عناية نظام الجملة الإنجليزية، وخمسة عشر في تحديد أدوات العطف المناسبة. وترجم الأغلبية من 
N. M. Mohammed Rizvi, Munas M.H.A, Iqbal Saujan

الطلبة الجملة الخامسة صحيحا في درجة أعلى، وأما الأقلية منهم ثلاثة فأخطأوا في عناية نظام الجملة الإنجليزية، وثمانية في قواعد النحوية، واثنا عشر في تحديد أدوات العطف المناسبة، وتسعة في استخدام الكلمات الإنجليزية المناسبة للكلمات العربية. ويوضحها الجدول رقم(2.9)، والشكل(2.9). الجدول 2.9 أخطاء في ترجمة الجمل العربية إلى اللغة الإنجليزية

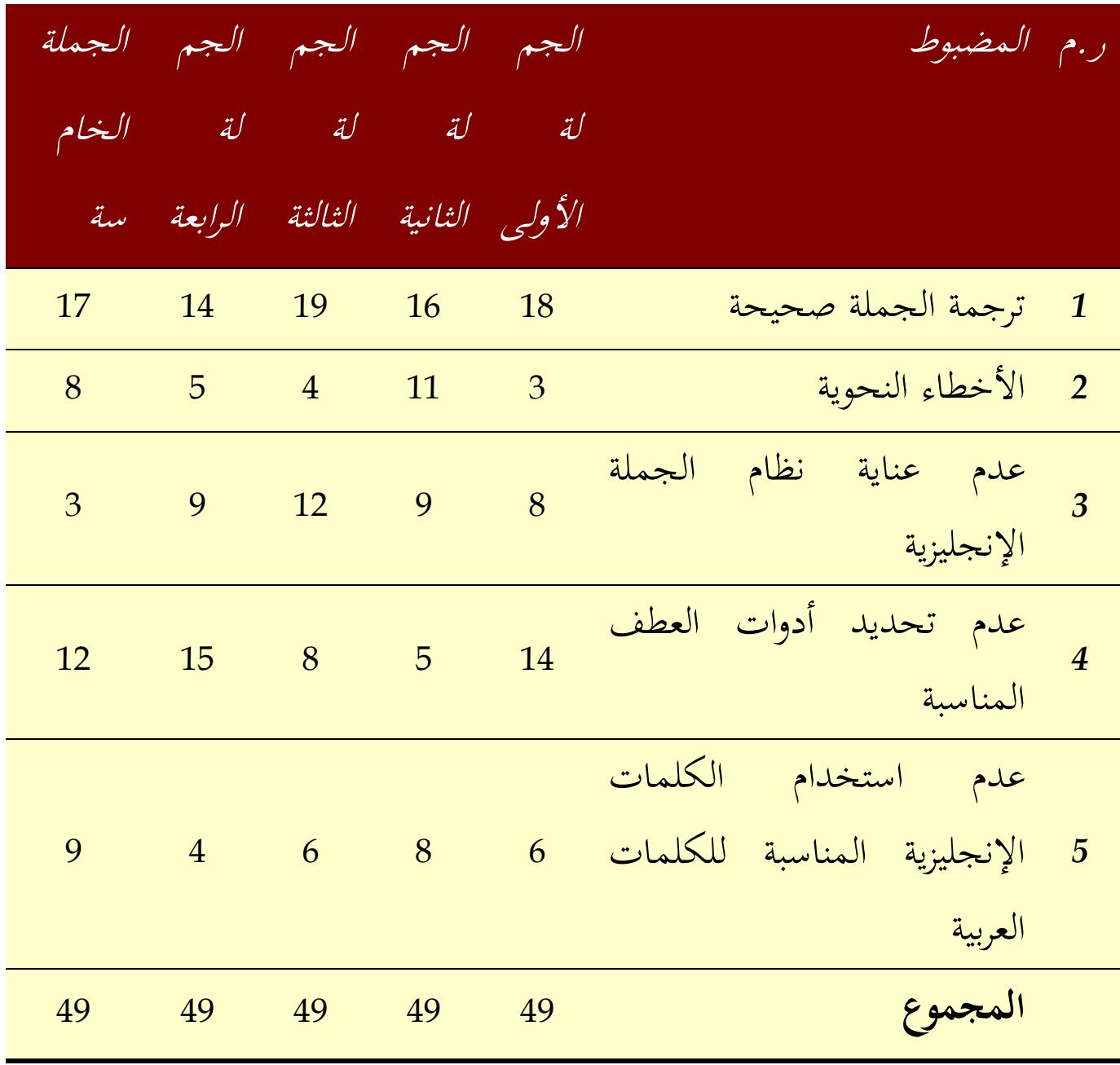

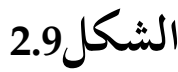


N. M. Mohammed Rizvi, Munas M.H.A, Iqbal Saujan

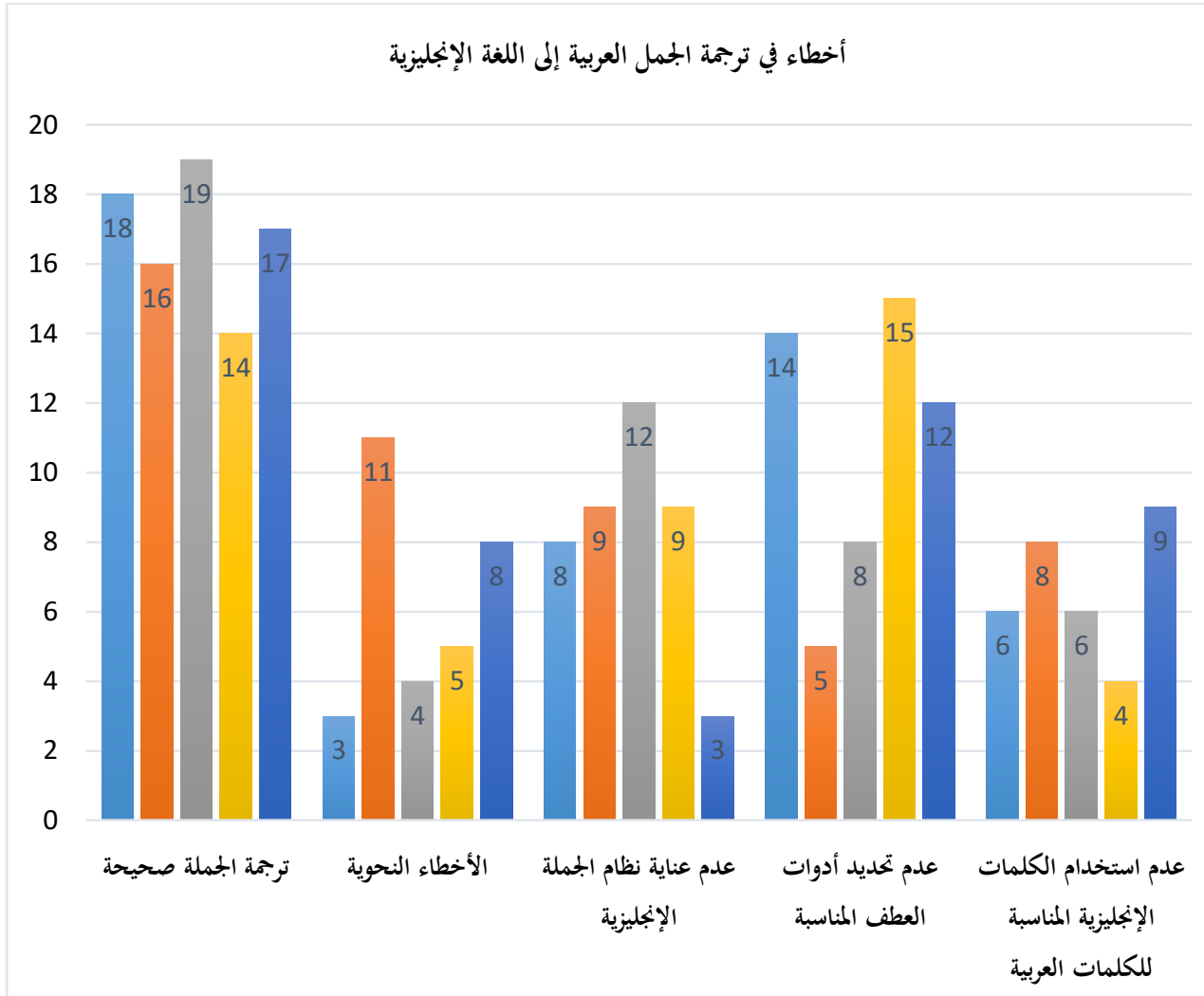

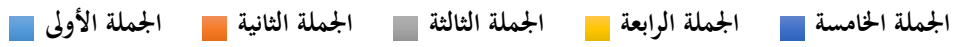

السؤال العاشر: وهو يعالج الصعوبات التي يواجهها الطلبة عند ترجمة هذه الجمل المذكورة إلى اللغة الإنجليزية وفق التدرج الرباعي ( موافق تماما، موافق، محايد، رافض تماما)، ويوضحها الجدول رقم(2.10).

الجدول 2.10 الصعوبات التي يواجهها عند ترجمة هذه الجمل العبية إلى اللغة الإنجليزية

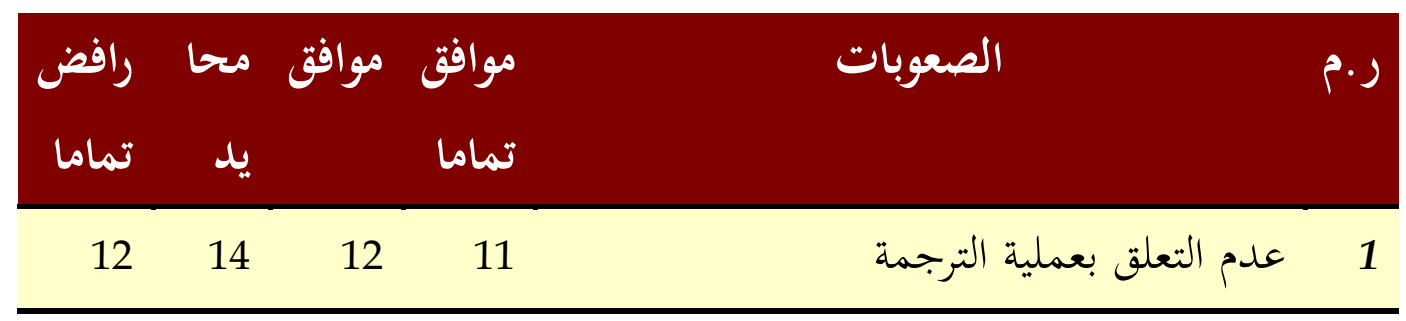


N. M. Mohammed Rizvi, Munas M.H.A, Iqbal Saujan

عدم وجود المعرفة عن قاعدة أدوات العطف $12 \quad 11 \quad 15 \quad 11 \quad 11$ في اللغة الإنجليزية

$\begin{array}{llll}11 & 15 & 13 & 10\end{array}$ 3

4 $10 \quad 14 \quad 15 \quad 10$

عدم التعلق بعملية الترجمة: واجه الأغلبية من الطلبة الصعوبات عند ترجمة الجمل العربية إلى اللغة الإنجليزية بسبب عدم التعلق بعملية الترجمة، وأربعة عشر حايدوا في وجود هذه القضية، أما الأقلية منهم فوافقوا فيه تماما، في حين اثنا عشر لم يقبلوا هذا السبب لمواجهة الصعوبات في الترجمة، ويوضحها الشكل رقم(2.10). الثكل 2.10

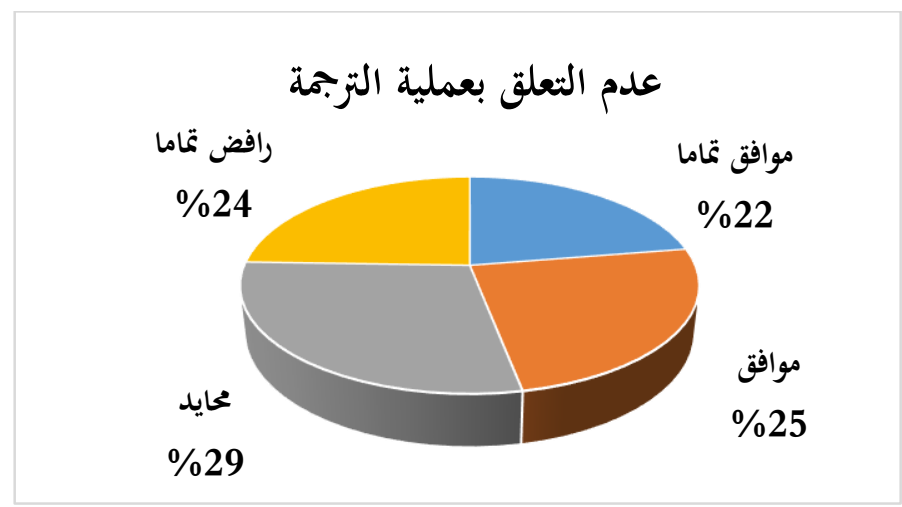

عدم وجود المعرفة عن قاعدة أدوات العطف في اللغة الإنجليزية: واجه اثنا عشر من الطلبة الصعوبات عند ترجمة الجمل العربية إلى اللغة الإنجليزية بسبب عدم وجود المعرفة عن قاعدة أدوات العطف في اللغة الإنجليزية ولكن أحد عشر وافقوا في وجود هذه القضية، أما الأقلية منهم أحد عشر فرفضوا فيه تماما، في حين 
N. M. Mohammed Rizvi, Munas M.H.A, Iqbal Saujan

خمسة عشر من الطلبة حايدوا هذا السبب لمواجهة الصعوبات في الترجمة، ويوضحها الشكل رقم (2.11).

\section{الشكل 2.11}

عدم وجود المعرفة عن قاعدة أدوات العطف في اللغة الإنجليزية

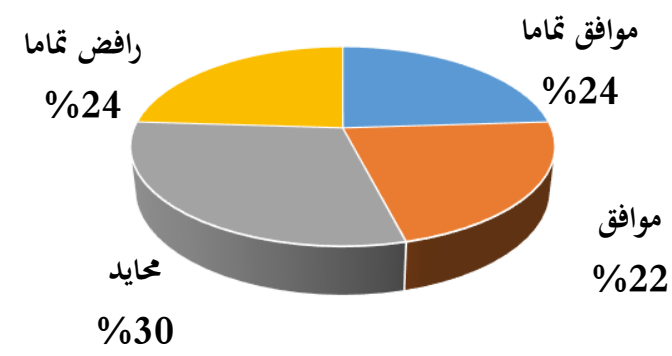

الضعف في الكتابة بالإنجليزية: واجه عشرة من الطلبة الصعوبات عند ترجمة الجمل العربية إلى اللغة الإنجليزية بسبب الضعف في الكتابة بالإنجليزية ولكن ثلاثة عشر وافقوا في وجود هذه القضية، أما الأغلبية منهم خمسة عشر فحايدوا فيه، في حين أحد عشر طالبا رفضوا هذا السبب تماما لمواجهة الصعوبات في الترجمة، ويوضحها الشكل رقم (2.12).

\section{الثكل 2.12}

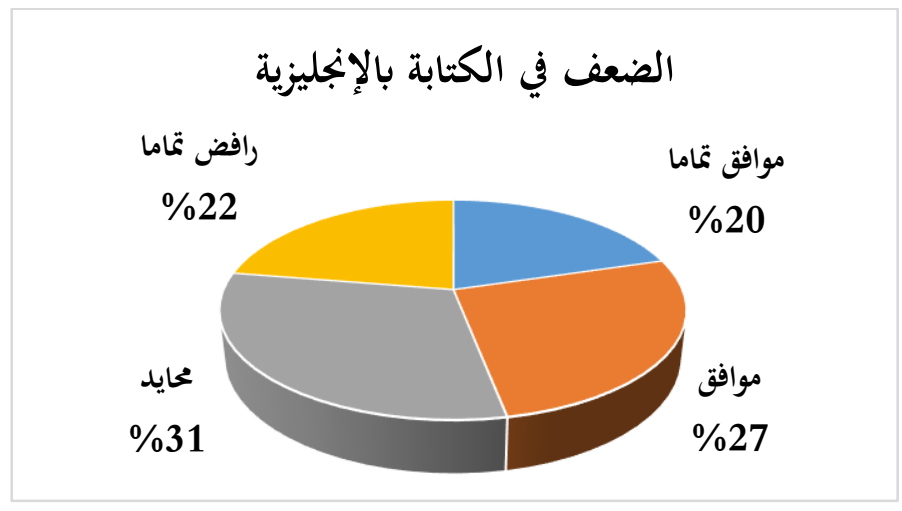


N. M. Mohammed Rizvi, Munas M.H.A, Iqbal Saujan

الضعف في إضافة الجمل في اللغة الإنجليزية: واجه عشرة من الطلبة الصعوبات عند ترجمة الجمل العربية إلى اللغة الإنجليزية بسبب الضعف في إضافة الجمل في اللغة الإنجليزية ولكن إثنا عشر وافقوا في وجود هذه القضية، أما الأغلبية منهم ستة عشر فحايدوا فيه، في حين أحد عشر من الطلبة رفضوا هذا السبب لمواجهة الصعوبات في الترجمة، ويوضحها الشكل رقم (2.13).

\section{الثكل 2.13}

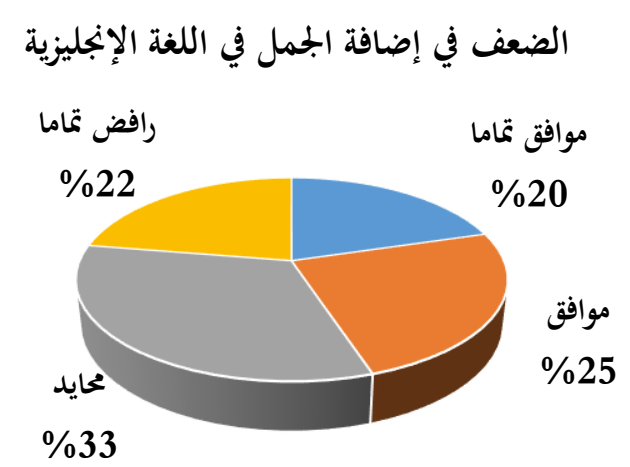

عدم معرفة استخدام أدوات العطف حسب السياق: واجه عشرة من الطلبة الصعوبات عند ترجمة الجمل العربية إلى اللغة الإنجليزية بسبب عدم معرفة استخدام أدوات العطف حسب السياق ولكن الأغلبية وافقوا في وجود هذه القضية، أما الأقلية منهم فرفضوا فيه تماما، في حين أربعة عشر من الطلبة حايدوا هذا السبب لمواجهة الصعوبات في الترجمة، ويوضحها الشكل رقم (2.14). الشكل 2.14 
N. M. Mohammed Rizvi, Munas M.H.A, Iqbal Saujan

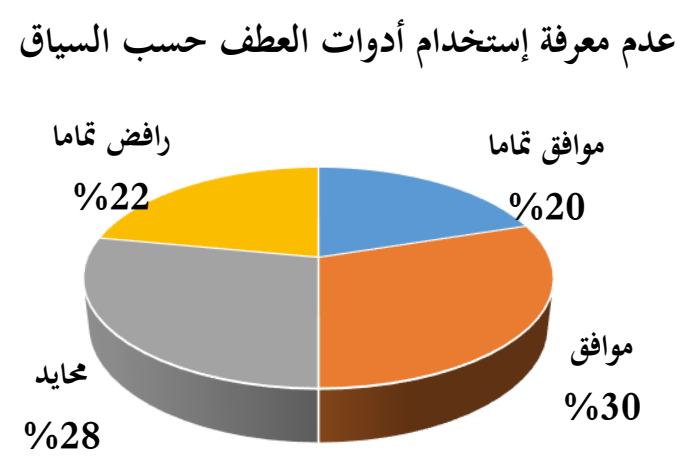

السؤال الحادي عشر: وهو يتضمن خمس جمل من اللغة الإنجليزية. ومن هذه الجمل، تحدد أخطاء الطلبة عند ترجمة الجمل الإنجليزية إلى اللغة العربية. John and Mary like rock music. You marry either Fathima or her sister. I will stay with you till you get better Ibrahim did not come, but his brother. Do you want the black one or the blue one? ترجم الأغلبية من الطلبة الجملة الأولى صحيحا في درجة أعلى، وأما الأقلية منهم فأخطأوا في قواعد النحوية، وثمانية منهم في عناية نظام الجملة العربية، وعشرة في تحديد أدوات العطف المناسبة، وستة في استخدام الكلمات العربية المناسبة للكلمات الإنجليزية. وترجم الأغلبية من الطلبة الجملة الثانية صحيحا في درجة أعلى، وأما الأقلية منهم فأخطأوا في تحديد أدوات العطف المناسبة، وتسعة في قواعد النحوية، وتسعة في عناية نظام الجملة العربية، وثمانية في استخدام الكلمات العربية المناسبة للكلمات الإنجليزية.

وترجم الأغلبية من الطلبة الجملة الثالثة صحيحا في درجة أعلى، وأما الأقلية

منهم فأخطأوا في قواعد النحوية، وفي استخدام الكلمات العربية المناسبة للكلمات 
N. M. Mohammed Rizvi, Munas M.H.A, Iqbal Saujan

الإنجليزية أيضا، وعشرة في عناية نظام الجملة العربية، وثمانية في تحديد أدوات العطف المناسبة. وترجم الأغلبية من الطلبة الجملة الرابعة صحيحا في درجة أعلى، وأما الأقلية منهم سبعة فأخطأوا في قواعد النحوية، وفي استخدام الكلمات العربية المناسبة للكلمات الإنجليزية أيضا، وتسعة في عناية نظام الجملة العربية، وثمانية في تحديد أدوات العطف المناسبة. وترجم الأغلبية تسعة عشر من الطلبة الجملة الخامسة صحيحا في درجة أعلى، وأما الأقلية منهم خمسة فأخطأوا في عناية نظام الجملة العربية، وثمانية في قواعد النحوية، وفي تحديد أدوات العطف المناسبة أيضا، وتسعة في استخدام الكلمات العربية المناسبة للكلمات الإنجليزية، ويوضحها

$$
\text { الجدول رقم (2.11)، والشكل رقم (2.15). }
$$

\section{الجدول 2.11 أخطاء في ترجمة الجمل الإنجليزية إلى اللغة العبية}

\begin{tabular}{|c|c|c|c|c|c|c|}
\hline الجملة & 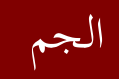 & 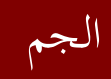 & الجم - | - الجم & 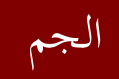 & المضبوط & קי \\
\hline 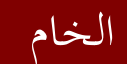 & لة & لة & لة & لة & & \\
\hline 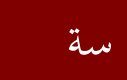 & 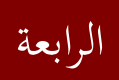 & 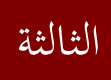 & 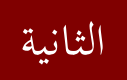 & الأول & & \\
\hline & & & & $\checkmark$ & & \\
\hline 19 & 16 & 19 & 18 & 20 & ترجمة الجملة صحيحة & 1 \\
\hline 8 & 7 & 6 & 9 & 5 & الأخطاء النحوية & 2 \\
\hline 5 & 9 & 10 & 9 & 8 & عدم عناية نظام الجملة العربية & 3 \\
\hline 8 & 8 & 8 & 5 & 10 & عدم المناسبة & 4 \\
\hline
\end{tabular}


N. M. Mohammed Rizvi, Munas M.H.A, Iqbal Saujan

\begin{tabular}{|c|c|c|c|c|c|}
\hline 9 & 7 & 6 & 8 & 6 & المناسبة استخدام الكلمات العربية \\
\hline 49 & 49 & 49 & 49 & 49 & المجموع \\
\hline
\end{tabular}

الشكل 2.15 
N. M. Mohammed Rizvi, Munas M.H.A, Iqbal Saujan

\section{أخطاء في ترجمة الجمل الإنجليزية \\ إلى اللغة العربية}

25

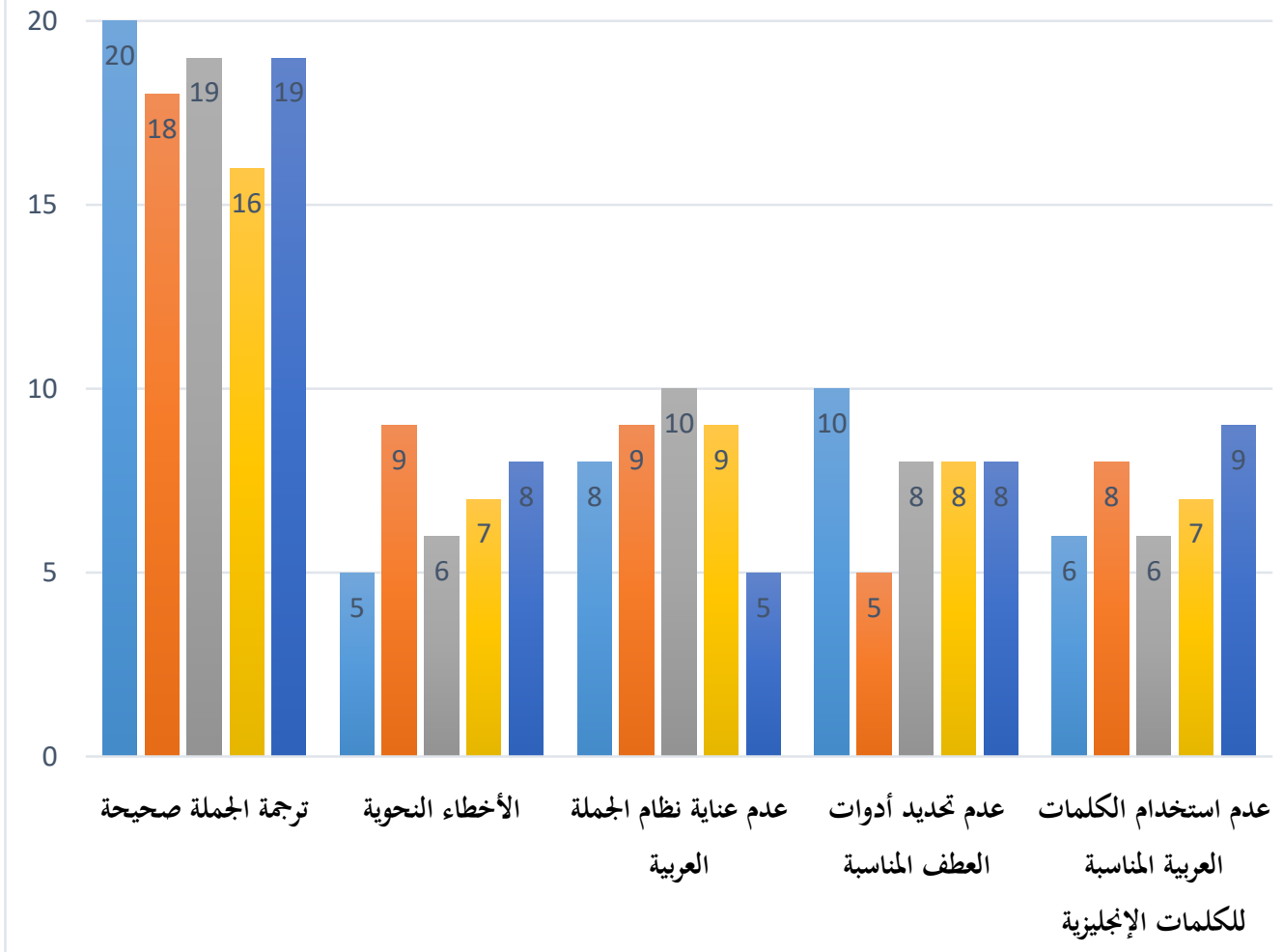

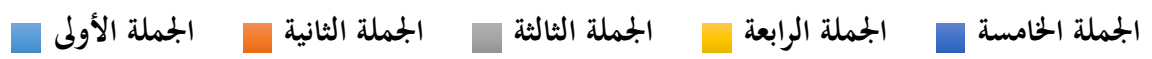

السؤال الثاني عشر : وهو يعالج الصعوبات التي يواجهها الطلبة عند ترجمة هذه

الجمل المذكورة إلى العربية وفق التدرج الرباعي (موافق تماما، موافق، محايد، رافض

تماما)، ويوضحها الجدول رقم (2.12) 
N. M. Mohammed Rizvi, Munas M.H.A, Iqbal Saujan

الجدول 2.12 الصعوبات التي يواجهها عند ترجمة هذه الجمل الإنجليزية إلى اللغة العربية

\begin{tabular}{|c|c|c|c|c|c|}
\hline ترافض & محا & موافة & توافق & الصعوبات & p.s \\
\hline 12 & 14 & 12 & 11 & عدم التعلق بعملية الترجمة & 1 \\
\hline 11 & 15 & 11 & 12 & 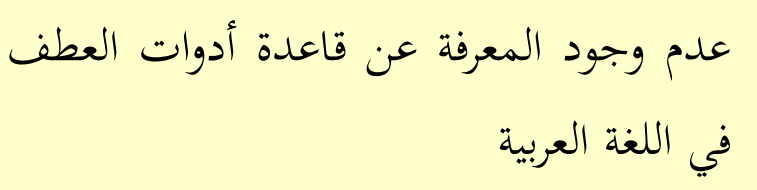 & 2 \\
\hline 11 & 15 & 13 & 10 & الضعف في الكتابة بالعربية & 3 \\
\hline 11 & 16 & 12 & 10 & الضعف في إضافة الجمل في اللغة العربية & 4 \\
\hline 10 & 14 & 15 & 10 & عدم معرفة استخدام أدوات العطف حسب & 5 \\
\hline
\end{tabular}

عدم التعلق بعملية الترجمة: وافق الأقلية من الطلبة تماما الصعوبات عند

ترجمة الجمل الإنجليزية إلى اللغة العربية بسبب عدم التعلق بالترجمة، ووافقوا اثنا عشر فيه ولكن أربعة عشر حايدوا في وجود هذه القضية، في حين اثنا عشر من الطلبة رفضوا تماما هذا السبب لمواجهة الصعوبات في الترجمة، ويوضحها الشكل رقم (2.16).

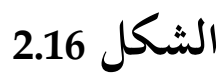


N. M. Mohammed Rizvi, Munas M.H.A, Iqbal Saujan

\section{عدم التعلق بعملية الترجمة}

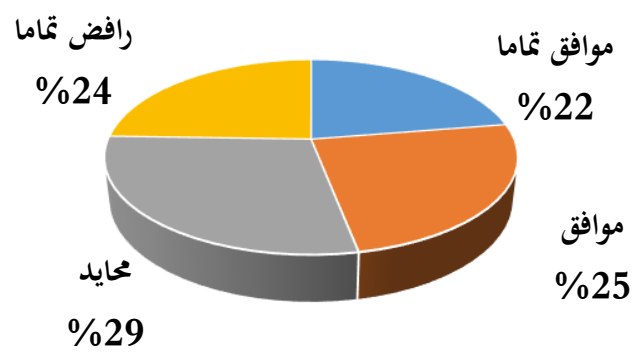

عدم وجود المعرفة عن قواعد أدوات العطف في اللغة العربية: وافق اثنا عشر من الطلبة الصعوبات عند ترجمة الجمل الإنجليزية إلى اللغة العربية بسبب عدم وجود المعرفة عن قاعدة أدوات العطف في اللغة العربية ولكن أحد عشر من الطلبة وافقوا في وجود هذه القضية، أما الأقلية منهم فرفضوا فيه تماما، في حين خمسة عشر من الطلبة حايدوا هذا السبب لمواجهة الصعوبات في الترجمة، ويوضحها الشكل رقم (2.17).

\section{الثكل 2.17}

عدم وجود المعرفة عن قاعدة أدوات العطف في اللغة العربية

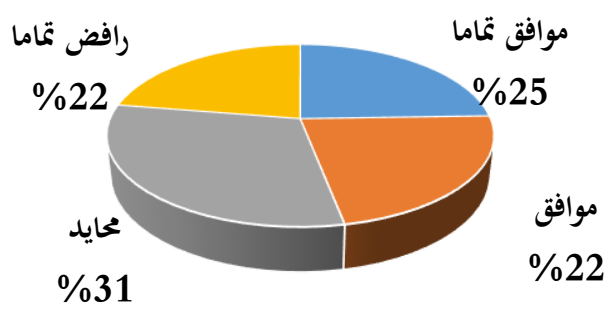

الضعف في الكتابة بالعربية: وافق عشرة من الطلبة الصعوبات عند ترجمة الجمل الإنجليزية إلى اللغة العربية بسبب الضعف في الكتابة بالعربية ولكن ثلاثة عشر وافقوا فيه، أما الأغلبية منهم خمسة عشر فحايدوا فيه، في حين أحد عشر 
N. M. Mohammed Rizvi, Munas M.H.A, Iqbal Saujan

من الطلبة رفضوا هذا السبب تماما لمواجهة الصعوبات في الترجمة، ويوضحها الشكل رقم(2.18).

\section{الثكل 2.18}

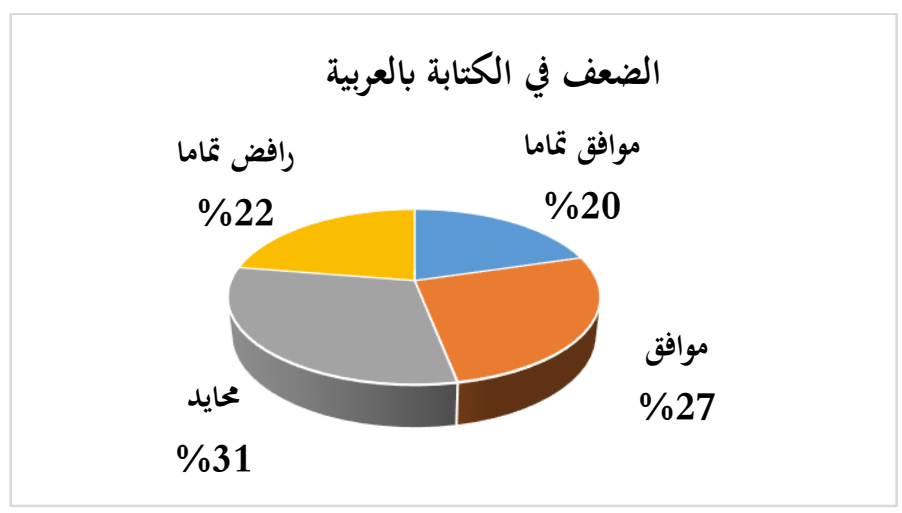

الضعف في إضافة الجمل في اللغة العربية: وافق عشرة تماما من الطلبة الصعوبات عند ترجمة الجمل العربية إلى اللغة الإنجليزية بسبب الضعف في إضافة الجمل في اللغة الإنجليزية ولكن اثنا عشر من الطلبة وافقوا في وجود هذه القضية،

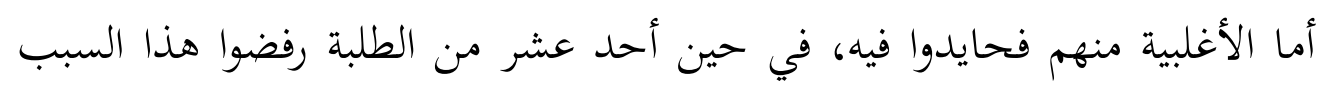
تماما لمواجهة الصعوبات في الترجمة، ويوضحها الشكل رقم(2.19). الشكل 2.19

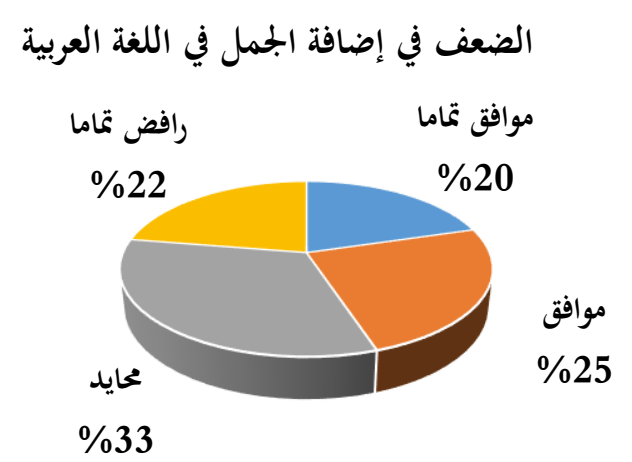


N. M. Mohammed Rizvi, Munas M.H.A, Iqbal Saujan

عدم معرفة استخدام أدوات العطف حسب السياق: وافق عشرة من الطلبة الصعوبات عند ترجمة الجمل العربية إلى اللغة الإنجليزية بسبب عدم معرفة استخدام أدوات العطف حسب السياق ولكن خمسة عشر وافقوا في وجود هذه القضية، أما الأغلبية منهم فحايدوا فيه، في حين عشرة من الطلبة رفضوا هذا السبب لمواجهة الصعوبات في الترجمة، ويوضحها الشكل رقم (2.20). الشكل 2.20

\section{عدم معرفة إستخدام أدوات العطف حسب السياق}

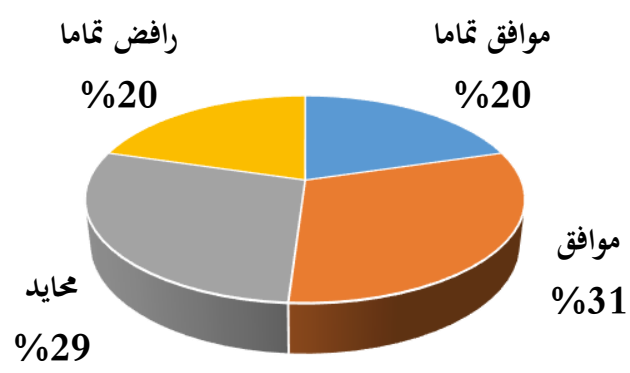

يتناول هذا الموضوع النتائج لتحليل البيانات من الإستبانات والمقابلات الشخصية التي تهاف قياس الصعوبات التي يواجهها الطلبة عند ترجمة أدوات العطف بين اللغتين العربية والإنجليزية. ويعالج تحليل البيانات حول موضوعين رئيسين. هما: الصعوبات في أدوات العطف بين اللغتين العربية والإنجليزية، والأسباب التي تؤدي إلى هذه الصعوبات عند ترجمة الجمل بين اللغتين العربية والإنجليزية.

\section{الصعوبات والأخطاء في ترجمة أدوات العطف بين اللغتين العربية والإنجليزية}

يوضح تحليل الإستبانات أن الطلبة الذين يتعلمون اللغتين العربية والإنجليزية يواجهون الصعوبات والأخطاء عند ترجمة أدوات العطف بين اللغتين العربية 
N. M. Mohammed Rizvi, Munas M.H.A, Iqbal Saujan

والإنجليزية، في حين أنهم يواجهون الصعوبات عند ترجمة أدوات العطف من اللغة العربية إلى اللغة الإنجليزية أكثر من الصعب عند ترجمة الجمل من اللغة الإنجليزية إلى اللغة العربية. عندما ترجم الطلبة من اللغة العربية إلى اللغة الإنجليزية فهم واجهوا الصعوبات لأسباب شتى ومنها: عدم التعلق بعملية الترجمة، عدم وجود المعرفة عن قواعد استخدام أدوات العطف في اللغة الإنجليزية، والضعف في الكتابة باللغة الإنجليزية، والضعف في إضافة الجمل في اللغة الإنجليزية، وعدم معرفة استخدام أدوات العطف حسب السياق. وعندما ترجم الطلبة من اللغة الإنجليزية إلى اللغة العربية فهم واجهوا الصعوبات لأسباب شتى ومنها: عدم التعلق بعملية الترجمة، وعدم وجود المعرفة عن قاعدة أدوات العطف في اللغة العربية، والضعف في الكتابة باللغة العربية، والضعف في إضافة الجمل في اللغة العربية، وعدم معرفة استخدام أدوات العطف حسب السياق.

أما الأخطاء التي وقعت من الطلبة عند الترجمة من اللغة العربية إلى اللغة الإنجليزية فهي :الأخطاء النحوية، وعدم عناية نظام الجملة الإنجليزية، وعدم تحديد أدوات العطف المناسبة، وعدم استخدام الكلمات الإنجليزية المناسبة للكلمات العربية، وأما الأخطاء النحوية وعدم استخدام الكلمات الإنجليزية المناسبة للكلمات العربية فوقعت أكثر من غيرها. أما الأخطاء النحوية فهي تحدث من التعريف والتنكير، والضمائر، وحروف الجر، وحروف النفي أي أن الطلبة قد استخدموا الأدوات في الأمكنة غير المناسبة وفي الأشكال المتغيرة المختلفة. أما الأخطاء التي وقعت من الطلبة عند الترجمة من اللغة الإنجليزية إلى اللغة العربية فهي :الأخطاء النحوية، وعدم استخدام الكلمات العربية المناسبة للكلمات الإنجليزية، وعدم عناية 
N. M. Mohammed Rizvi, Munas M.H.A, Iqbal Saujan

نظام الجملة العربية، وعدم تحديد أدوات العطف المناسبة، وأن الأخطاء النحوية وعدم تحديد أدوات العطف المناسبة وقعت أكثر من غيرها.

قد حدد الباحث الأسباب التي أدت إلى الصعوبات والأخطاء في ترجمة أدوات العطف بين اللغتين العربية والإنجليزية وهي قلة الرغبة في استخدام اللغتين العربية والإنجليزية صحيحا، قلة القراءة في اللغتين الإنجليزية والعربية، عدم الحرص في اطلاع اللغتين العربية والإنجليزية، التعلم حسب حاجات العصر، عدم وجود المعرفة عن قواعد اللغتين العربية والإنجليزية، عدم وجود المعرفة تماما عن قاعد أدوات العطف في اللغتين العربية والإنجليزية، عدم التعلق بعملية الترجمة، قلة التدريبات والتطبيقات في الترجمة. قلة ثروة الكلمات، و عدم وجود المعرفة عن السياق اللغوي.

الخلاصة

إن الباحث قد بذل جهوده خلال صفحات هذا البحث في إلقاء الضوء على معرفة الصعوبات والأخطاء عند ترجمة أدوات العطف بين اللغتين العربية والإنجليزية. أما الفصل الأول فيتحدث عن أطروحة البحث، هي يتضمن من المقدمة، والمشكلة، والأهداف، والأسئة، والأهمية، والحدود، ومنهج البحث والدراسات السابقة. والفصل الثاني يبحث في تحليل الإستبانة ونتائجها تحت أربعة أبواب : أما الباب الأول فيقدم طريقة إجراءات الدراسات الميدانية، والثاني يحلل الإستبانات وتفسيرها، والثالث يفسر النتائج من تحليل الدراسات الميدانية، والرابع 
N. M. Mohammed Rizvi, Munas M.H.A, Iqbal Saujan

يقدم خاتمة البحث مع التركيز على نتائج البحث والتوصيات التي يجب الإلتزام بها عند ترجمة أدوات العطف في اللغتين العربية والإنجليزية، وذلك لمعالجة مشكلة هذا البحث.

قد حصل الباحث على النتائج الآتية خلال هذه الدراسة إن تعليم اللغة العربية

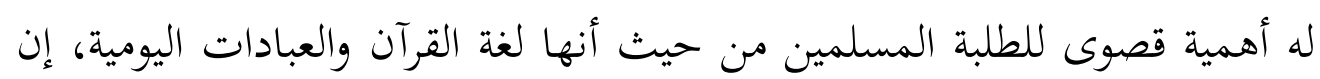
للطلبة علاقة وثيقة باللغة العربية من حيث أنهم يتعلمونها من المدارس العربية والجامعات. وهم يتعلمونها لحصول شهادة البكاليوريوس، إن اللغة العربية واللغة الإنجليزية تنتميان إلى العائلتين اللغويتين المختلفتين، أما اللغة العربية فتنتمي إلى اللغات السامية مهما اللغة الإنجليزية تنتمي إلى جرمانية. حسب هذه القضية، أنهما تختصان نظاما مستقلا حسب مكوناتهم اللغوية. بناء على هذا، أن هناك يكون أوجه الخلاف أكثر من أوجه الشبه، لم يتقن الطلبة ترجمة أدوات العطف في اللغتين العربية والإنجليزية مهما يتكلموا اللغتين العربية والإنجليزية، إن الطلبة يحسنون في ترجمة الجمل بين اللغتين العربية والإنجليزية، ولكنهم لم يعرفوا تماما عن كيفية إضافة الجمل بأدوات العطف المناسبة، تقع الأخطاء في قواعد أدوات العطف بسبب قلة القراءة في اللغتين الإنجليزية والعربية، وإن الطلبة يعرفون بعض أدوات العطف ولكنهم لم يعرفوا عن كيفية استخدامها حسب سياق الجمل.

\section{المصادر والمراجع}

Al-Farahidi, A.A. (1992). al'Ayn. Cairo: Al-Hilal Library House.

Al-Farra, A.Z. (1993). Maani al'Qur'an. Egypt: The Egyptian House of Composition and Translation. 
N. M. Mohammed Rizvi, Munas M.H.A, Iqbal Saujan

Al-Mubarrad,A.A (1997). al'Muqtadab. Beirut: The World of Books Ibn Manzur. (1994). Lisanul Arab. Beirut: Dar Sader.

Al-Fayoumi, (1999). al-Misbahul Munir fi Gharib al-Sharh al-Kabir. Beirut: Modern Library.

Al-Suyuti, J. (1998). Hameul Hawamie fi Sharh Jame al'Jawamie. Beirut: Scientific Books House.

al-Mawsili, I.Y. (2001). Sharh al'Mufasal. Beirut: Scientific Books House. Ibn Malik, J. (1995). Sharhul Kafiat al'Shaafia. Dar Al-Mamoun Heritage. Ibn Asfour, A.M.A. (1996). Sharh Jumal al'Zujajii. Beirut: Scientific Books House.

Ibn Al-Sarraj, A.B. (1998). al'Usul fi al'Nahw. Beirut: Al-Resala Foundation.

Hassan, A. (1997). al'Nahw al'Wafi. Cairo: Dar al-Maarif.

Ibn Malik, J.A. (1998). Tashil al'Fawayid watakmil al'Maqasid. Beirut: Scientific Books House, 1998.

Al-Subki, A.K. (1999). Al'iibhaj fi Sharh al'Minhaj. Beirut: Scientific Books House.

Ibn Al-Anbari. (1992). Asrar al'Arabia. Damascus: Arab Scientific Academy Publications.

Ibn Hisham, J.A (1993). Awdah al'masalik 'iilaa 'alfiat ibn Malik. Beirut: Modern Library. 